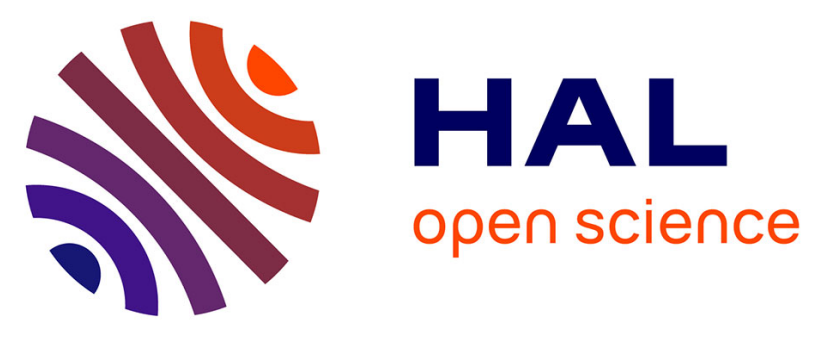

\title{
Modelling Salmonella transmission among pigs from farm to slaughterhouse: Interplay between management variability and epidemiological uncertainty
}

Jordi Ferrer Savall, Caroline Bidot, Mily Leblanc-Maridor, Catherine Belloc, Suzanne Touzeau

\section{To cite this version:}

Jordi Ferrer Savall, Caroline Bidot, Mily Leblanc-Maridor, Catherine Belloc, Suzanne Touzeau. Modelling Salmonella transmission among pigs from farm to slaughterhouse: Interplay between management variability and epidemiological uncertainty. International Journal of Food Microbiology, 2016, 229, pp.33-43. 10.1016/j.ijfoodmicro.2016.03.020 • hal-01334840

\author{
HAL Id: hal-01334840 \\ https://hal.inria.fr/hal-01334840
}

Submitted on 25 Nov 2016

HAL is a multi-disciplinary open access archive for the deposit and dissemination of scientific research documents, whether they are published or not. The documents may come from teaching and research institutions in France or abroad, or from public or private research centers.
L'archive ouverte pluridisciplinaire HAL, est destinée au dépôt et à la diffusion de documents scientifiques de niveau recherche, publiés ou non, émanant des établissements d'enseignement et de recherche français ou étrangers, des laboratoires publics ou privés.

\section{(ㅇ)(1) $\$$}

Distributed under a Creative Commons Attribution - NonCommercial - NoDerivatives 44.0 


\title{
Modelling Salmonella transmission among pigs from farm to slaughterhouse: interplay between management variability and epidemiological uncertainty
}

\author{
Jordi Ferrer Savall ${ }^{*, a}$, Caroline Bidot ${ }^{\mathrm{a}}$, Mily Leblanc-Maridor ${ }^{\mathrm{b}}$, Catherine \\ Belloc $^{\mathrm{b}}$, Suzanne Touzeau ${ }^{\mathrm{c}, \mathrm{d}}$ \\ ${ }^{a}$ MaIAGE, INRA, Université Paris-Saclay, 78350 Jouy-en-Josas, France \\ ${ }^{b}$ INRA, LUNAM Université, Oniris, UMR 1300 BioEpAR, 44300 Nantes, France \\ ${ }^{c} I N R A$, Univ. Nice Sophia Antipolis, CNRS, UMR 1355-7254 Institut Sophia Agrobiotech, \\ 06900 Sophia Antipolis, France \\ ${ }^{d}$ BIOCORE, Inria, 06900 Sophia Antipolis, France
}

\begin{abstract}
Salmonella carriage and cutaneous contamination of pigs at slaughter are a major risk for carcass contamination. They depend on Salmonella prevalence at farm, but also on transmission and skin soiling among pigs during their journey from farm to slaughterhouse. To better understand and potentially control what influences Salmonella transmission within a pig batch during this transport and lairage step, we proposed a compartmental, discrete-time and stochastic model. We calibrated the model using pork chain data from Brittany. We carried out a sensitivity analysis to evaluate the impact of the variability in management protocols and of the uncertainty in epidemiological parameters on three model outcomes: prevalence of infection, average cutaneous contamination and number of new infections at slaughter. Each outcome is mainly influenced by a single management factor: prevalence at slaughter mainly depends on the prevalence at farm, cutaneous contamination on the contamination of lairage pens and new infections on the total duration of transport and lairage. However, these results are strongly affected by the uncertainty in epidemiological parameters. Reexcretion of carriers due to stress does not have a major impact on the number
\end{abstract}

\footnotetext{
${ }^{*}$ Corresponding author

Email address: jordi.ferrer.savall@gmail.com (Jordi Ferrer Savall)
}

Preprint submitted to International Journal of food Microbiology 
of new infections.

Key words: stochastic model, sensitivity analysis, transport and lairage, salmonellosis, swine

\section{Introduction}

Human salmonellosis is the second most common foodborne zoonosis in the European Union (EU) and it is frequently attributed to the consumption of pork products (Hald et al., 2003, Pires and Hald, 2010). According to surveil-

5 lance estimates by the European Food Safety Authority (EFSA), Salmonella is endemic in the pig population: around $10 \%$ of pigs at slaughter have infected Jymph nodes and around $8 \%$ of the processed carcasses are contaminated (European Food Safety Authority, 2008). In order to reduce the incidence of human salmonellosis, EU states are required to monitor each stage of the pork supply chain and urged to adopt control strategies ensuring pig health and welfare, depending on their country-specific pig industry, herd statuses, slaughterhouse structures and compliance with hygienic measures (EFSA Panel on Biological Hazards (BIOHAZ), 2010).

Non-negligible transmission rates during pig transport and lairage were reported, both among animals belonging to the same herd and across herds (Hurd et al. 2001a b). Transmission occurs primarily via the fecal-oral route: a healthy pig gets infected after ingesting a large number of microorganisms. In turn, infected individuals intermittently excrete large numbers of the bacteria in their faeces, contaminating their local environment. Stress imposed by food withdrawal, transportation, or lairage can significantly increase the number of shedding pigs, as well as the amount of both excreted and ingested Salmonella (Scherer et al., 2008).

There is strong evidence showing that prevalence at slaughter depends on (i) the proportion of animals shedding at departure from farm (Boughton et al. 25 2007), (ii) the degree of environmental contamination and (iii) the duration of exposure to this contamination (Hurd et al., 2001a; Mannion et al., 2012). How- 
ever, there is a wide diversity in the epidemiological status of pigs departing from farms (European Food Safety Authority, 2008), in the transport conditions and in the exposure to environmental contamination (Rostagno et al. 2003), both 30 at an individual level and at a batch level (Hernández et al., 2013). Moreover, many epidemiological characteristics of Salmonella spread remain highly uncertain, for instance: the dose-response relationship between the environmental contamination and the infection probability of healthy pigs (Boughton et al. 2007, Loynachan and Harris, 2005), the excretion rate of shedders (Ivanek et al.

35 2012 Martín-Peláez et al., 2009, Tanaka et al., 2014), or the rate of stressed non-shedding carriers reverting to excretion (Scherer et al., 2008). This prevents drawing definite conclusions regarding the relative impact of factors on the risk of carcass contamination (Rostagno and Callaway, 2012).

The complex interplay between biological and management processes affect40 ing Salmonella transmission appeals for a modelling approach to evaluate the impact of different factors at different levels of the production chain Hotes et al. 2012, Smid et al. 2012). Mechanistic models have been successfully implemented at a farm level (Berriman et al., 2013, Hill et al., 2015; Lurette et al. 2008). Transport and lairage are usually not represented, with the no-

45 table exception of an EFSA scientific report (VLA, DTU, RIVM, 2010), which performed a quantitative microbial risk analysis of the pork production chain to investigate the effect of interventions at different points of the food chain (Schaffner and Doyle, 2008). It resulted in a hierarchy of control measures and an estimation of their impact on the public health risk of salmonellosis. The model describing transmission during transport and lairage was recently detailed (Simons et al., 2015).

In line with these communications, an exhaustive exploration of the interactions between management conditions and epidemiological settings in a batch during transport and lairage was carried out. The aim of this study was to ${ }_{55}$ assess their relative impact on the epidemiological status of pigs at slaughter under different transmission regimes, while considering the internal carriage and cutaneous contamination, as both can lead to (cross-)contamination during 
slaughter. The impact of the slaughter processes on carcass contamination is outside the scope of this study.

\section{Material and methods}

\subsection{Model description}

This work presents a discrete-time stochastic epidemiological model that follows a single batch from farm to slaughter considering three stages: waiting at farm (stage $F$ ), transport by truck (stage $T$ ) and waiting at lairage (stage $A$ ), as shown in Figure 1 1 . The time spent in each stage is given by the stage duration $t_{X}$. The batch is characterised by its epidemiological state $B(t)$ at time $t$, describing the number of healthy $(S)$, latent $(E)$, actively shedding $(I)$ and non-shedding carrier $(R)$ pigs (Figure $1 \mathrm{~b}$ ), and by its average cutaneous contamination $C(t)$, which represents the average skin soiling of a pig. Its initial state at time $t_{0}=0$ is defined by three parameters: batch size $b_{0}=$ $S\left(t_{0}\right)+E\left(t_{0}\right)+I\left(t_{0}\right)+R\left(t_{0}\right)$, which remains constant over time, Salmonella prevalence at farm $p_{0}=\frac{I\left(t_{0}\right)+R\left(t_{0}\right)}{b_{0}}$ and initial cutaneous contamination $c_{0}$. At each stage $X \in\{F, T, A\}$, pens are characterised by their final environmental contamination $Q_{X}$, initialised by $q_{X}$.

The model considers three stochastic processes governing the evolution of the variables from the beginning $\left(t_{b, X}\right)$ to the end $\left(t_{e, X}=t_{b, X}+t_{X}\right)$ of each stage: excretion, transmission and skin soiling (Figure 1 $1 \mathrm{~b}$ ). Note that the beginning of the waiting at farm corresponds to the initial time $\left(t_{b, F}=t_{0}=0\right)$ and that stages are connected without lapses (for instance, $t_{e, F}=t_{b, T}$ ).

To determine the final environmental contamination $Q_{X}$, the amount of Salmonella shed by active shedders using random samples taken from a normal distribution $(\mathcal{N})$ was computed with:

$$
Q_{X}=q_{X}+\mathcal{N}\left(\varepsilon, \frac{\varepsilon}{10}\right) I\left(t_{b, X}\right) t_{X}
$$

where $\varepsilon$ is the excretion rate. The standard deviation of the excretion rate is set arbitrarily to $\varepsilon / 10$, so that the different levels of $\varepsilon$ explored in the sensitivity 

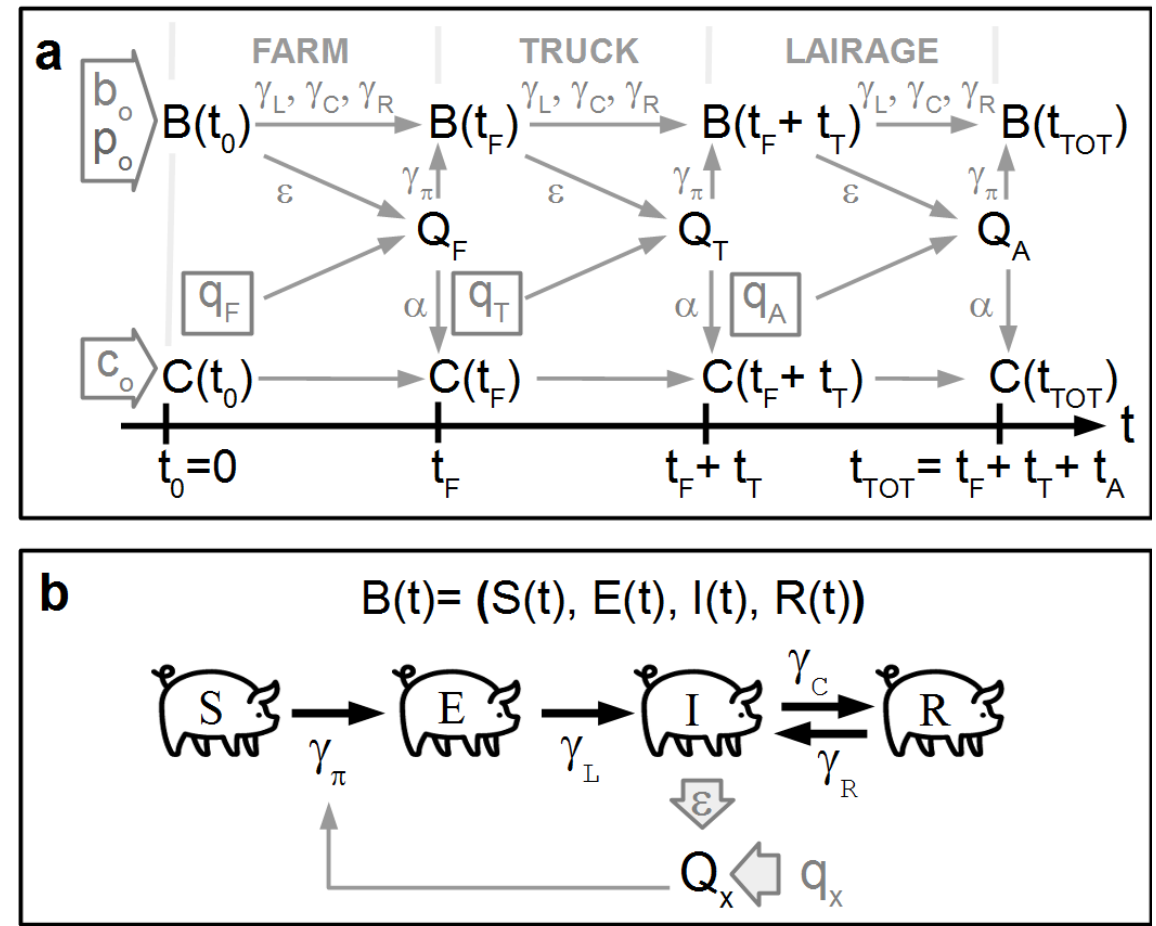

Figure 1: Outline of the stochastic compartmental model representing Salmonella dynamics in a batch from farm to slaughter. (a) The model derives the environmental contamination $\left(Q_{X}\right)$, the epidemic state $(B)$ and the average cutaneous contamination $(C)$ of the batch at each stage $\left(X: F=\right.$ farm,$T=$ transport and $A=$ lairage), from the batch size $\left(b_{0}\right)$, prevalence at farm $\left(p_{0}\right)$, initial cutaneous contamination $\left(c_{0}\right)$ and environmental contaminations $\left(q_{X}\right)$; it considers Salmonella excretion $(\varepsilon)$, contamination $\left(\gamma_{\pi}\right)$, infection dynamics $\left(\gamma_{L}, \gamma_{C}\right.$ and $\left.\gamma_{R}\right)$ and skin soiling $(\alpha)$. (b) The epidemic model considers four infection states $(S=$ healthy, $L=$ latent, $I=$ active shedder and $C=$ non-shedder carrier); fecal-oral transmission $\left(\gamma_{\pi}\right)$ is driven by environmental contamination $\left(Q_{X}\right)$, resulting from the initial contamination $\left(q_{X}\right)$ and from shedding $(\varepsilon)$; the other transition rates between states $\left(\gamma_{L}, \gamma_{C}\right.$ and $\left.\gamma_{R}\right)$ only depend on the stage duration $\left(t_{X}\right)$. 
analysis do not overlap. Occasional samples of negative numbers, which very seldom occurred, were replaced by zero.

In the following equations, we dropped the stage subscript $X$ for $t_{X, b}$ and $t_{X, e}$, as there was no possible ambiguity. For each stage, the model updates the number of pigs in each epidemiological state (Figure 1b) as follows:

$$
\left\{\begin{array}{l}
S\left(t_{e}\right)=S\left(t_{b}\right)-N_{S \rightarrow E}\left(t_{b}, t_{e}\right) \\
E\left(t_{e}\right)=E\left(t_{b}\right)+N_{S \rightarrow E}\left(t_{b}, t_{e}\right)-N_{E \rightarrow I}\left(t_{b}, t_{e}\right) \\
I\left(t_{e}\right)=I\left(t_{b}\right)+N_{E \rightarrow I}\left(t_{b}, t_{e}\right)-N_{I \rightarrow R}\left(t_{b}, t_{e}\right)+N_{R \rightarrow I}\left(t_{b}, t_{e}\right) \\
R\left(t_{e}\right)=R\left(t_{b}\right)+N_{I \rightarrow R}\left(t_{b}, t_{e}\right)-N_{R \rightarrow I}\left(t_{b}, t_{e}\right)
\end{array}\right.
$$

Each $N_{Y \rightarrow Z}$ corresponds to the number of pigs transiting from epidemiological state $Y$ to state $Z$ and was determined by a random sample drawn from a binomial distribution $(\mathcal{B})$ :

$$
\left\{\begin{array}{l}
N_{S \rightarrow E}=\mathcal{B}\left(S\left(t_{b}\right), \gamma_{\pi}\left(t_{e}-t_{b}\right)\right) \\
N_{E \rightarrow I}=\mathcal{B}\left(S\left(t_{b}\right), \gamma_{L}\left(t_{e}-t_{b}\right)\right) \\
N_{I \rightarrow R}=\mathcal{B}\left(S\left(t_{b}\right), \gamma_{C}\left(t_{e}-t_{b}\right)\right) \\
N_{R \rightarrow I}=\mathcal{B}\left(S\left(t_{b}\right), \gamma_{R}\left(t_{e}-t_{b}\right)\right)
\end{array}\right.
$$

where $\gamma_{\pi}, \gamma_{L}, \gamma_{C}$ and $\gamma_{R}$ are the transition probabilities from $S$ to $E$, from $E$ to $I$, from $I$ to $R$ and from $R$ to $I$, respectively; they depend on the stage duration ${ }_{95}\left(t_{e}-t_{b}\right)$.

The probabilities $\gamma_{L}=1-e^{\frac{-\left(t_{e}-t_{b}\right)}{\tau_{L}}}$ and $\gamma_{C}=1-e^{\frac{-\left(t_{e}-t_{b}\right)}{\tau_{C}}}$ were computed using the average durations of the latent period $\left(\tau_{L}\right)$ and of the shedding phase $\left(\tau_{C}\right)$ of Salmonella infection in pigs.

The probabilities $\gamma_{\pi}=1-(1-\pi)^{\left(t_{e}-t_{b}\right)}$ and $\gamma_{R}=1-(1-\rho)^{\left(t_{e}-t_{b}\right)}$ were computed by cumulating the infection $(\pi)$ and re-excretion $(\rho)$ rates over the stage duration (Figure 2 2 ). The infection and re-excretion rates are defined the probability for a pig to become infected and to start re-excreting, respectively, during a unit of time. Indeed, these individual probabilities are integrated values that would be easier to measure than proper continuous rates and that can well 

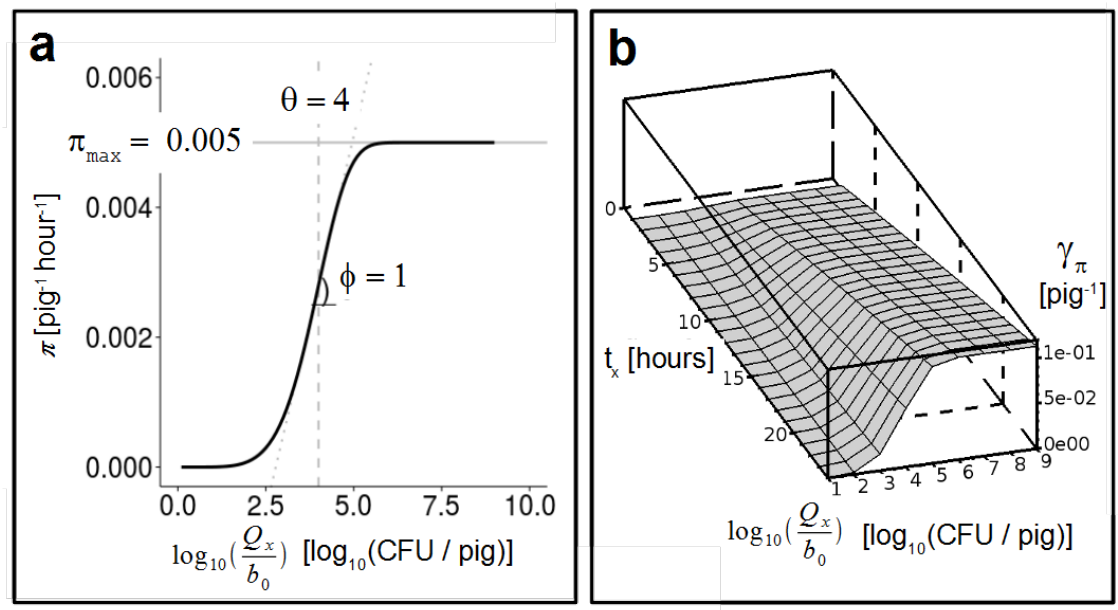

Figure 2: Representation of the functions implemented for the fecal-oral transmission at each stage ( $X: F=$ farm , $T=$ transport and $A=$ lairage). (a) The dose-response function computes the infection rate $(\pi)$, defined as the probability for a pig to become infected during a unit of time, as a function of the environmental contamination per pig $\left(Q_{X} / b_{0}\right)$; its parameters are the maximum probability at saturation $\left(\pi_{\max }\right)$, the contamination inflection point $(\theta)$ and the steepness of the curve $(\phi)$. (b) The probability of infection $\left(\gamma_{\pi}\right)$ cumulates the rate $(\pi)$ over the stage duration $t_{X}$. 
used in a discrete-time model.

The infection rate $\pi$ is given by the dose-response function, a Weibull cumulative distribution function of the logarithm of the environmental contamination $Q_{X}$ divided by the batch size $b_{0}$ (Figure 2 $\mathrm{p}$ ). This particular shape was chosen for its flexibility and efficiency in parameter exploration (Fekedulegn et al. 1999). Similar simulation results were obtained when testing other types of Sshaped curves, characterised by the same parameters: probability of infection under saturated environments $\left(\pi_{\max }\right)$, environmental contamination corresponding to an inflection point of the infection probability $(\theta)$ and steepness of the curve $(\phi)$. Pigs are not individualised in the model, which follows the number exposed to the same average environmental contamination $\left(\pi=\pi\left(Q_{X} / b_{0}\right)\right)$ is adequate in such a context. The re-excretion rate, which depends on the stress of pigs, was assumed constant from farm to slaughter.

Finally, the average cutaneous contamination of a pig $C(t)$ was computed from $Q_{X}$ using a fixed rate of skin soiling $\alpha$ :

$$
C\left(t_{e}\right)=C\left(t_{b}\right)+\alpha \frac{Q_{X}}{b_{0}} .
$$

Individual contaminations can be drawn from a probability distribution using this average cutaneous contamination as parameter.

We chose to concentrate on four model outcomes: the prevalence at slaughter $p_{s}=\frac{I\left(t_{\mathrm{TOT}}\right)+R\left(t_{\mathrm{TOT}}\right)}{b_{0}}$, the average cutaneous contamination at slaughter ${ }_{125} c_{s}=C\left(t_{\mathrm{TOT}}\right)$, the number of new infections during transport and lairage $N_{I}=S\left(t_{0}\right)-S\left(t_{\mathrm{TOT}}\right)$ and the number of pigs that revert from carriers to active shedders during transport and lairage $N_{R}=R\left(t_{0}\right)-R\left(t_{\mathrm{TOT}}\right)$.

\subsection{Model parameters}

To simulate the model, its parameters were either set to fixed values or they varied to take into account the variability, i.e. the "natural" variations observed among individuals or in the processes represented in the model, and the uncertainty, i.e. the lack of knowledge, in real data (Table 1). 


\begin{tabular}{|c|c|c|c|c|}
\hline & Parameter & Value(s) & Unit & Source \\
\hline$b_{0}$ & Batch size & 100 & pigs & (BDPORC, 2010) \\
\hline$c_{0}$ & Initial cutaneous contamination & $10^{1}$ & $\frac{\mathrm{CFU}}{\mathrm{pig}}$ & $*$ \\
\hline$\alpha$ & Proportion of skin soiling & 0.1 & - & * \\
\hline$\tau_{L}$ & Average latent period & 0.5 & hours & (Hurd et al. 2002) \\
\hline \multirow[t]{2}{*}{$\tau_{C}$} & Average shedding duration & $5 \times 10^{4}$ & hours & Boughton et al. 2007) \\
\hline & & Management factors & & \\
\hline$p_{0}$ & Prevalence at farm & $0,5,10,20$ & hours & VLA, DTU, RIVM 2010) \\
\hline$t_{\mathrm{TOT}}$ & Total duration & $8,16,24$ & hours & BDPORC, 2010) \\
\hline$t_{F}$ & Waiting time at farm & $2.5,5.5,9$ & hours & BDPORC, 2010) \\
\hline$t_{T}$ & Transport duration & $0.5,1.5,3$ & hours & BDPORC, 2010 \\
\hline$t_{A}$ & Waiting time at lairage & $5,9,12$ & hours & BDPORC, 2010) \\
\hline$q_{F}$ & Farm initial contamination & $0,10^{5}, 10^{6}, 10^{7}, 10^{9}$ & $\mathrm{CFU}$ & Frotin et al. 2007) † \\
\hline$q_{T}$ & Truck initial contamination & $0,10^{5}, 10^{6}, 10^{7}, 10^{9}$ & $\mathrm{CFU}$ & Frotin et al. 2007) $\dagger$ \\
\hline \multirow[t]{2}{*}{$q_{A}$} & Lairage initial contamination & $0,10^{5}, 10^{6}, 10^{7}, 10^{9}$ & $\mathrm{CFU}$ & Frotin et al. 2007) $\dagger$ \\
\hline & & Epidemiological factors & & \\
\hline$\varepsilon$ & Excretion rate & $10,10^{2}, 10^{3}, 10^{4}, 10^{5}$ & $\frac{\mathrm{CFU}}{\text { pig.hour }}$ & $\ddagger$ \\
\hline$\pi_{\max }$ & Infection rate at saturation & $0.002,0.005,0.01$ & $\frac{1}{\text { pig.hour }}$ & $\ddagger$ \\
\hline$\theta$ & Threshold contamination & $3,4,5 \mathrm{lc}$ & $\log _{10}\left(\frac{\mathrm{CFU}}{\mathrm{pig}}\right)$ & $\ddagger$ \\
\hline$\phi$ & Steepness of the dose-response curve & $0.1,0.5,1$ & $\frac{\mathrm{pig}^{-1} \text { hour }^{-1}}{\log _{10}\left(\frac{\mathrm{CFU}}{\mathrm{pig}}\right)}$ & $\ddagger$ \\
\hline$\rho$ & Re-excretion rate & $0.005,0.01,0.05,0.1,0.5$ & $\frac{1}{\text { pig.hour }}$ & $\ddagger$ \\
\hline
\end{tabular}

Table 1: Parameters of the model. The waiting at farm $\left(t_{F}\right)$, transport in truck $\left(t_{T}\right)$ and waiting at lairage $\left(t_{A}\right)$ durations were set from real data in Brittany (BDPORC, 2010). The total duration was computed as follows: $t_{\mathrm{TOT}}=t_{F}+t_{T}+t_{A}$. Initial prevalence $\left(c_{0}\right)$ of batches were set according to surveillance data (VLA, DTU, RIVM 2010). * Initial cutaneous contamination $\left(c_{0}\right)$ and proportion of skin soiling $(\alpha)$ where fixed to arbitrary values due to lack of experimental data. † Reference values for the range of contaminations that can be found in slaughterhouses in Brittany. They are consistent with other measurements found in the literature (Small et al. 2006 Swanenburg et al. 2001). $\ddagger$ Epidemiological factors were chosen to obtain model outcomes that were consistent with the literature (Boughton et al. 2007, Hurd et al. 2001a b): $\pi_{\max }, \theta$ and $\phi$ are the parameters of the dose-response function, $\tau_{L}$ is the lag before an infected pig starts shedding and $\tau_{C}$ is the shedding duration. 
The batch size $\left(b_{0}\right)$ was set to a fixed value representing an average size of batches. This choice does not affect our results because transmission is not modeled at an individual level. The initial cutaneous contamination $\left(c_{0}\right)$ and proportion of skin soiling $(\alpha)$ were fixed to arbitrary values due to lack of information. In particular, we considered that all pigs were initially clean. The relative impact of management and epidemiological parameters on skin soiling does not depend on these parameter values because, they affect neither excretion nor transmission. The latent period $\left(\tau_{L}\right)$ and the shedding duration $\left(\tau_{C}\right)$ were fixed because their variations had no impact on the outcomes: the former is much shorter and the latter much longer than the transport and lairage durations.

To deal with the management factor variability, we chose three levels of total duration $\left(t_{\mathrm{TOT}}=t_{F}+t_{T}+t_{A}\right)$, corresponding to the 5 th percentile, median and 95th percentile of the distribution of the waiting at farm $\left(t_{F}\right)$, transport $\left(t_{T}\right)$ and lairage $\left(t_{A}\right)$ times provided by the professional union BDPORC (BDPORC, 2010). We explored four levels of prevalence at farm $\left(p_{0}\right)$ and five levels of initial environmental contamination $\left(q_{X}\right)$ at each stage, extracted from data describing the pork industry in Brittany (Frotin et al., 2007).

Because of the uncertainty on the epidemiological factors, we first screened 2500 combinations of epidemiological parameters and pre-selected the parameters generating outcomes in accordance with the literature (Hurd et al., 2001a b). This ensured that our model could reproduce realistic patterns. We then selected 27 discrete combinations of dose-response parameters $\left(3 \pi_{\max } \times 3 \theta \times 3 \phi\right)$, 5 levels of the excretion rates $(\varepsilon)$ and 5 levels of the re-excretion rate $(\rho)$.

\subsection{Model exploration and sensitivity analyses}

Our model considers three sources of randomness. Firstly, transmission is a stochastic process as pigs under same transport and lairage conditions may or may not become contaminated. Secondly, management contexts (initial status of batches and transport conditions) are diverse. Thirdly, parameters describing Salmonella spread are highly uncertain. From now on, we use the term scenario 
to refer to a combination of management contexts $\left(q_{X}\right.$ at each stage, $p_{0}$ and $\left.t_{\mathrm{TOT}}=t_{F}+t_{T}+t_{A}\right)$ and epidemiological settings $\left(\pi_{\max }, \theta, \phi, \rho\right.$ and $\left.\varepsilon\right)$.

To determine the number of repetitions of each scenario that capture stochasticity in transmission, we carried out a preliminary analysis varying this number in $\{51,101,201,501,801,1001,5001\}$. We found that $N_{\text {reps }}=501$ repetitions were sufficient to ensure that model outcomes do not differ from their asymptotic distributions: the variance of the mean of each model outcome remained fixed at around $1 \%$ when 500 or more repetitions were considered. The mean of each model outcome was calculated by averaging its value among the repetitions. The variance of the mean was computed by repeating 100 times the calculation of the mean of the model outcome (100 different samples).

Intra-scenario and inter-scenario variabilities were compared, revealing that the former was significantly smaller than the latter for all three outcomes. Therefore, we decided to examine inter-scenario effects on the model outcomes averaged over intra-scenario repetitions.

Two numerical experiments were carried out to systematically explore the impact of the variability of management contexts and of the uncertainty in epidemiological parameters on the model outcomes $\left(p_{s}, c, N_{I}\right.$ and $\left.N_{R}\right)$, averaged over $N_{\text {reps }}=501$ repetitions. Firstly, all pigs initially infected at farm were considered as active shedders, by setting $I(0)=p_{0} b_{0}$ and hence $R(0)=0$. As the average shedding duration $\tau_{C}$ is very long compared to the total duration $t_{\mathrm{TOT}}$ (Table 1), the impact of the re-excretion rate $\rho$ was ignored, hence obtaining a 185 simplified $S \rightarrow E \rightarrow I$ model. This entailed exploring 202500 scenarios combining 1500 management contexts and 135 epidemiological settings. Secondly, all pigs initially infected at farm were considered as non-shedding carriers, by setting $R(0)=p_{0} b_{0}$ and hence $I(0)=0$. Even with a initial population consiting of non-shedding carriers, the impact of $\rho$ could not be neglected, so the original ${ }_{190} S \rightarrow E \rightarrow I \leftrightarrow R$ model was maintained. However, the impact of the steepness of the dose-response curve $\phi$ was now overlooked, as it turned out to be the least significant factor in the previous experiment. As a result, 337500 scenarios, combining 1500 management contexts and 225 epidemiological settings were 
explored in this second set of simulations.

A global sensitivity analysis to explore the influence of the scenario factors on the model outcomes (Saltelli et al., 2000) was performed by carrying out out a standard ANOVA for each outcome considering up to second-order interactions. The sensitivity index associated with each term was evaluated, split into main effect of a factor and two-factor interactions, as the ratio between the sum of squares corresponding to that term and the total sum of squares. The total sensitivity index was computed for each factor as the sum of the sensitivity indices corresponding to this factor (main effect plus interactions involving the factor). The sensitivity indices obtained quantify the fraction of outcome variance among simulations explained by the variation of each factor within its value range.

All simulations and analyses were performed using $\mathrm{R}$ and the multisensi package (Lamboni et al., 2011).

\section{Results}

\subsection{Inter-scenario sensitivity analysis without carriers (SEI model)}

The relative impact of management and epidemiological factors on batches initially composed of healthy pigs and active shedders is assessed in Figures 3 and 4 .

Outcomes of the scenarios explored in this first experiment exhibit wide asymetric distributions (Figure $3 \mathrm{a}-\mathrm{c}$ ) that aggregate overlapping distributions even when results are split by the factors that most impact each variable (Figure $3 \mathrm{~d}-$ f).

There are generally new infections during transport and lairage: the $95 \%$ confidence interval of this outcome is $C I_{0.95}\left(N_{I}\right)=[0,22]$ pigs (Figure 3 ;,f). Prevalence may increase from its initial value $p_{0} \in[0,20] \%$ to its value at slaughter $C I_{0.95}\left(p_{s}\right)=[0,32.7] \%$ (Figure $3 \mathrm{~d}$ ). The average cutaneous contamination saturates under highy contaminated environments, regardless of the infection dynamics (Figure 3p). 

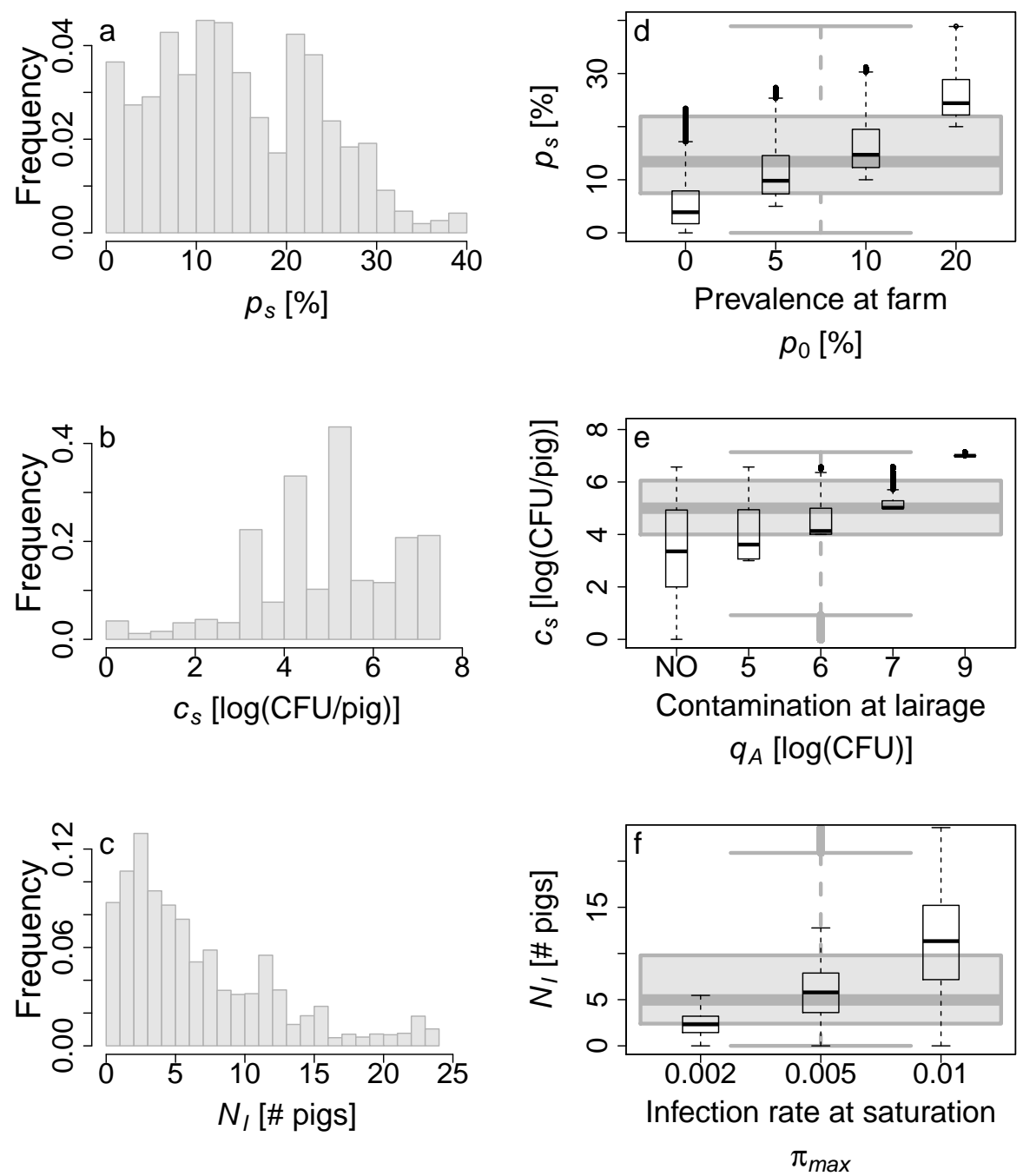

Figure 3: Inter-scenario distributions of the SEI model outcomes: (a,d) prevalence at slaughter, $(\mathbf{b}, \mathbf{e})$ average cutaneous contamination at slaughter and $(\mathbf{c}, \mathbf{f})$ new infections from farm to slaughter. Histograms $(\mathbf{a}-\mathbf{c})$ present the model outcomes averaged over 501 repetitions for 202500 scenarios. Scenarios cross 27 dose-response functions with 5 excretion rates and 1500 management contexts. Corresponding boxplots $(\mathbf{d}-\mathbf{f})$ are presented alongside (grey), as well as boxplots split by the values of the factor that most affects the outcome considered (white): (d) initial prevalence at farm, (e) environmental contamination at lairage and (f) maximum infection probability at saturation (parameter of the dose-response function). 
(I) All factors
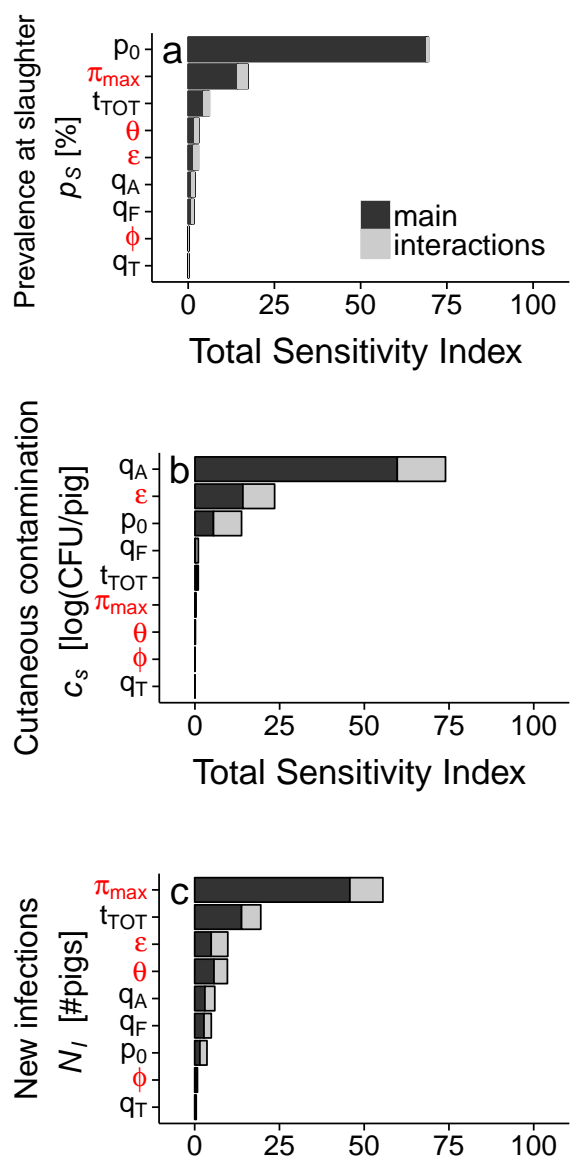

Total Sensitivity Index
(II) Management factors
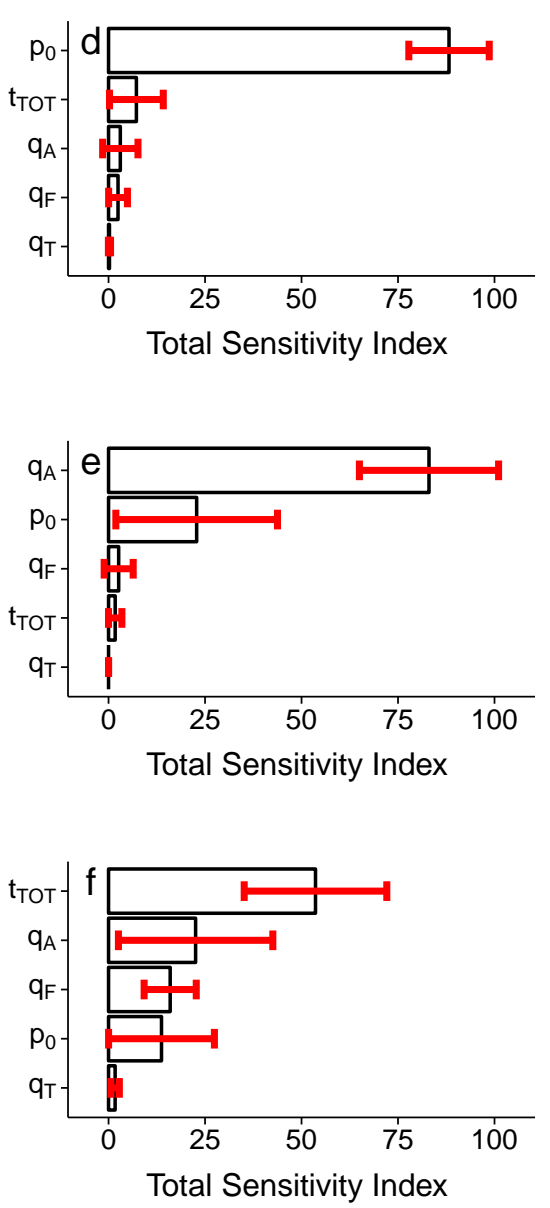

Figure 4: Inter-scenario sensitivity analysis of the $S E I$ model outcomes: (a,d) prevalence at slaughter, $(\mathbf{b}, \mathbf{e})$ average cutaneous contamination at slaughter and $(\mathbf{c}, \mathbf{f})$ new infections from farm to slaughter. Outcomes are averaged over 501 repetitions for 202500 scenarios that cross 27 dose-response functions with 5 excretion rates and 1500 management contexts. Column (I): total sensitivity indices for all factors, split into main effect (dark grey) and interactions (light grey). Column (II): total sensitivity indices for the management factors alone, averaged over all scenarios sharing the same management constraints; so the associated errorbars represent the variability arising from the uncertainty over the epidemiological parameters. Factors are described in Table 1 
For all outcomes, there is always a factor explaining more than $50 \%$ of the inter-scenario variability, whereas the interactions between factors explain less transmissibility affects the relative impact of the initial contamination of lairage 

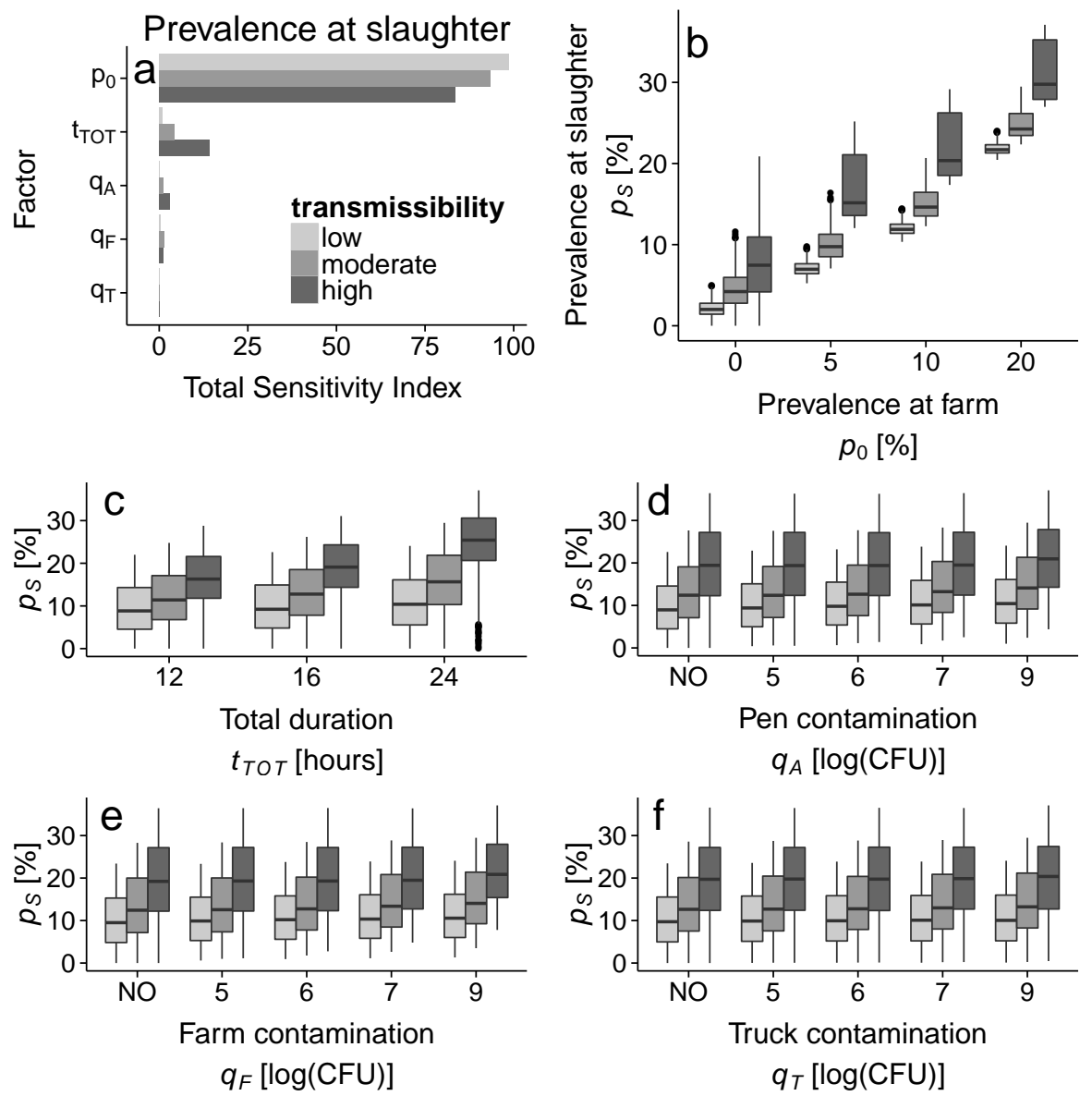

Figure 5: Detailed analysis of the prevalence at slaughter $\left(p_{s}\right)$ for three epidemiological settings: low (light grey), moderate (medium grey) and (high (dark grey) transmissibilities. $p_{s}$ is averaged over 501 repetitions for 1500 scenarios (management contexts) of the SEI model. (a) Total sensitivity indices for the management factors. (b-f) Boxplots split by factor values, in decreasing order of importance. Factors are described in Tables 1 and 2 

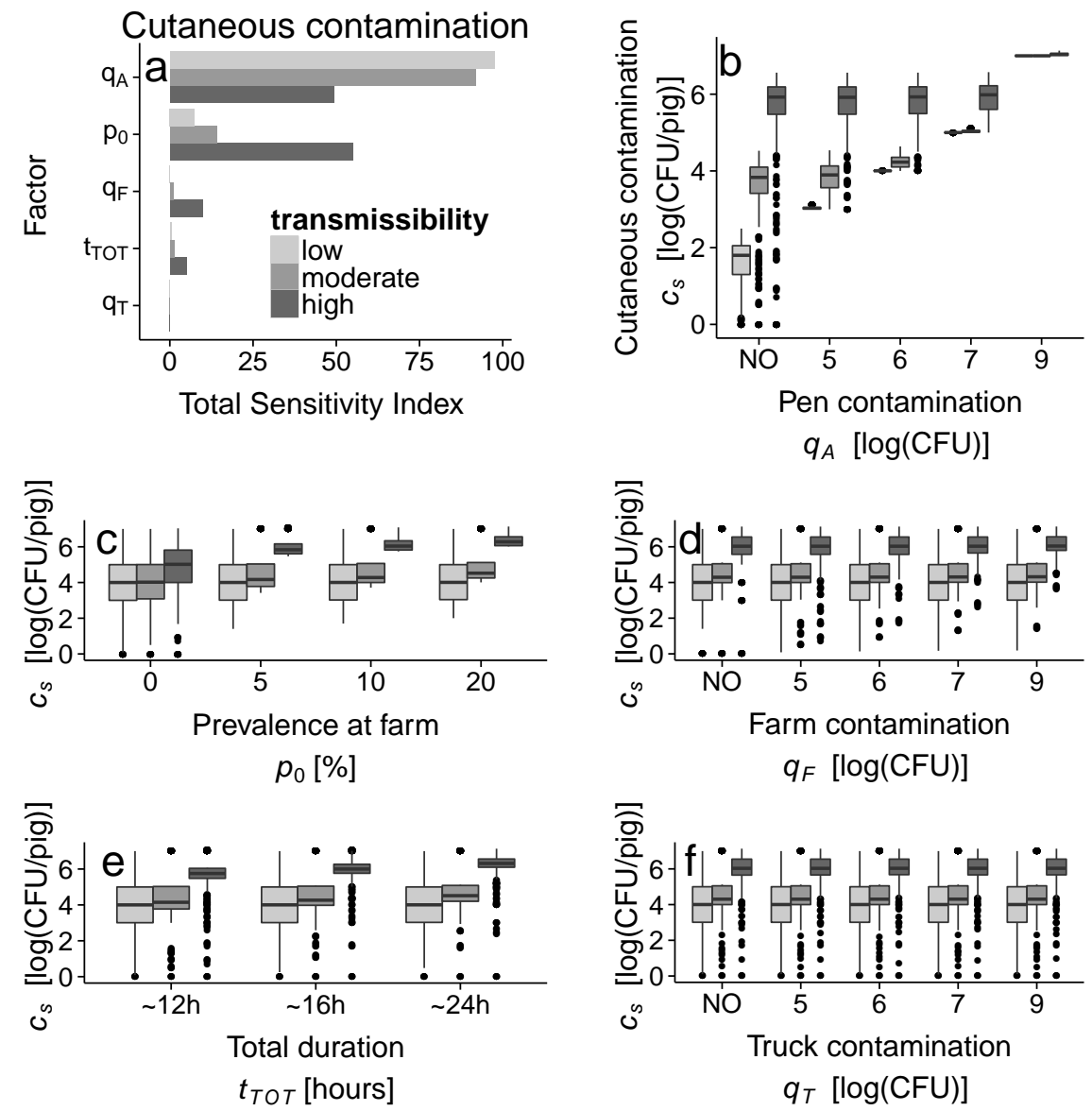

Figure 6: Detailed analysis of the average cutaneous contamination at slaughter $\left(c_{s}\right)$ for three epidemiological settings: low (light grey), moderate (medium grey) and (high (dark grey) transmissibilities. $c_{s}$ is averaged over 501 repetitions for 1500 scenarios (management contexts) of the SEI model. (a) Total sensitivity indices for the management factors. (b-f) Boxplots split by factor values, in decreasing order of importance. Factors are described in Tables 1 and 2 


\begin{tabular}{lcccc}
\hline Parameter & Low & Moderate & High & Unit \\
\hline$\varepsilon$ & $10^{2}$ & $10^{4}$ & $10^{6}$ & $\frac{\mathrm{CFU}}{\text { pig.hour }}$ \\
$\pi_{\max }$ & 0.002 & 0.005 & 0.01 & $\frac{1}{\text { pig.hour }}$ \\
$\theta$ & 5 & 4 & 3 & $\log _{10}\left(\frac{\mathrm{CFU}}{\mathrm{pig}}\right)$ \\
$\phi$ & 0.1 & 0.5 & 1 & $\frac{\mathrm{pig}^{-1} \text { hour }^{-1}}{\log _{10}\left(\frac{\mathrm{CFU}}{\mathrm{pig}}\right)}$ \\
\hline
\end{tabular}

Table 2: Epidemiological parameters selected for the low, moderate and high transmissibilities. $\pi_{\max }$ : infection probability at saturation, $\theta$ : threshold contamination triggering infection, $\phi$ : steepness of the dose-response curve and $\varepsilon$ : excretion rate.

pens $\left(q_{A}\right)$ : this factor is determining under low transmissibility regimes but has less impact for higher transmissibility regimes (Figure 6a). In contrast, the relative impact of the initial prevalence at farm $\left(p_{0}\right)$ is more important under high transmissibility regimes. Secondly, there is a threshold for $q_{A}$ above which the average cutaneous contamination saturates and is no longer affected by other factors (Figure 6b). This threshold increases with transmissibility: for the low, moderate and high transmissibility regimes, saturation occurs for $q_{A} \geqslant 10^{5} \frac{\mathrm{CFU}}{\mathrm{pig}}$, $q_{A} \geqslant 10^{7} \frac{\mathrm{CFU}}{\mathrm{pig}}$ and $q_{A} \geqslant 10^{9} \frac{\mathrm{CFU}}{\mathrm{pig}}$, respectively.

Figure 7 shows that, even if the total duration $\left(t_{\mathrm{TOT}}\right.$ is the most important factor determining the number of new infections from farm to slaughter, the relative impact of the other factors is probably affected by the transmissibility regime. Under a high transmissibility, the second most important factor is the initial prevalence at farm $\left(p_{0}\right)$, while under low and moderate transmissibility regimes it is the initial contamination of lairage pens $\left(q_{A}\right)$.

\subsection{Impact of re-excretion (SEIR model)}

To explore the impact of re-excretion, all initial infected pigs were considered as non-shedding carriers. We analysed how the re-excretion rate $(\rho)$ alters the relative impact of management and epidemiological factors on the three previous model outcomes $\left(p_{s}, c_{s}\right.$ and $\left.N_{I}\right)$ and on the number of pigs reverting to excretion $\left(N_{R}\right)$.

This numerical experiment showed that the outcome distributions are more 

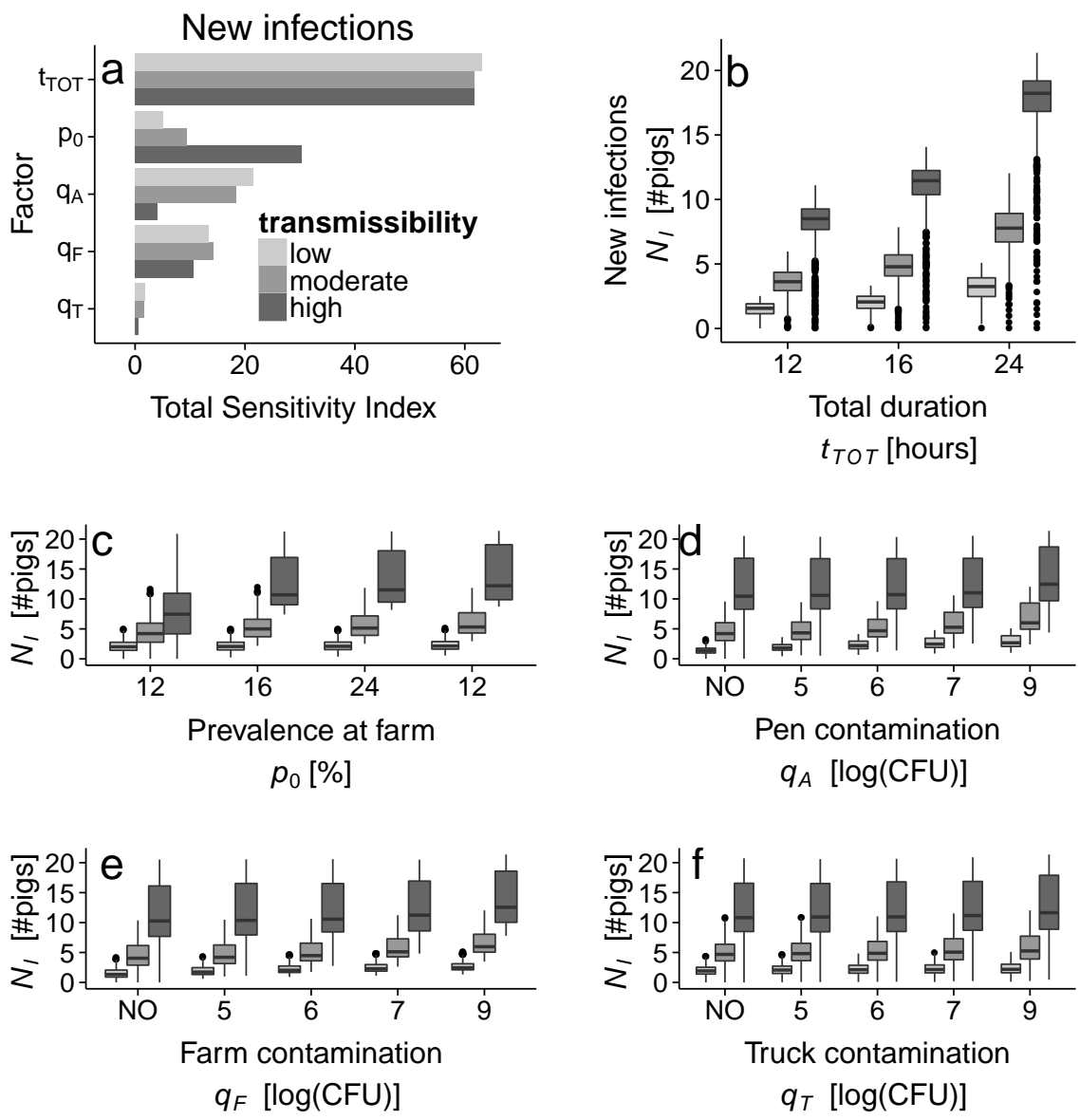

Figure 7: Detailed analysis of the new infections from farm to slaughter $\left(N_{I}\right)$ for three epidemiological settings: low (light grey), moderate (medium grey) and high (dark grey) transmissibilities. $N_{I}$ is averaged over 501 repetitions for 1500 scenarios (management contexts). (a) Total sensitivity indices for the management factors. (b-f) Boxplots split by factor values, in decreasing order of importance. Factors are described in Tables 1 and 2 
strongly shaped by the initial conditions. For instance, the distribution of the prevalence at slaughter $\left(p_{s}\right)$ clearly exhibits four peaks, corresponding to the four initial values of the prevalence at farm $\left(p_{0}\right)$ (Figure $\left.8 \mathrm{a}, \mathrm{e}\right)$.

Not surprisingly, we found that the outcomes of the SEIR model are lower than those of the SEI model (Table 3). Indeed, all initially infected pigs in the $S E I R$ model were non-shedding carriers. Not all became shedders and when they did, they globally started shedding later than in the $S E I$ model, in which all initially infected pigs were active shedders.

\begin{tabular}{lccc}
\hline Outcome & $C I_{0.95}(S E I)$ & $C I_{0.95}(S E I R)$ & Unit \\
\hline$p_{s}$ & {$[0.6,32.7]$} & {$[0.2,24.6]$} & $\%$ \\
$c_{s}$ & {$[1,7]$} & {$[1,6.3]$} & {$\left[\log _{10}\left(\frac{\mathrm{CFU}}{\mathrm{pig}}\right)\right]$} \\
$N_{I}$ & {$[0,22]$} & {$[0,16]$} & pigs \\
$N_{R}$ & $\mathrm{n} / \mathrm{a}$ & {$[0,20]$} & pigs \\
\hline
\end{tabular}

Table 3: Comparison of the model outcome distributions when all initial infected pigs are either active shedders (SEI model, 202500 scenarios) or non-shedding carriers (SEIR model, 337500 scenarios). The $95 \%$ confidence interval $\left(C I_{0.95}\right)$ is given for the prevalence at slaughter $\left(p_{s}\right)$, the average cutaneous contamination at slaughter $(c)$, the number of new infections from farm to slaughter $\left(N_{I}\right)$ and the number of infected pigs reverting to excretion from farm to slaughter $\left(N_{R}\right)$.

The re-excretion rate $(\rho)$ has a small impact on the model outcomes, except for $N_{R}$, which could be expected (Figure 9). It just explains around $10 \%$ of the variability for $c_{s}$ and less than $1 \%$ of the variability for $p_{s}$ and $N_{I}$. Introducing re-excretion affects the relative impact of the other factors by enhancing the effect of the initial conditions. For instance, the initial prevalence at farm $p_{0}$ and the initial contamination of the lairage pen $q_{A}$ have a greater impact on $p_{s}$ and $c_{s}$, respectively.

\section{Discussion}

We presented a stochastic model of Salmonella infection dynamics from farm to slaughter and we used it to analyse the impact of epidemiological and man- 

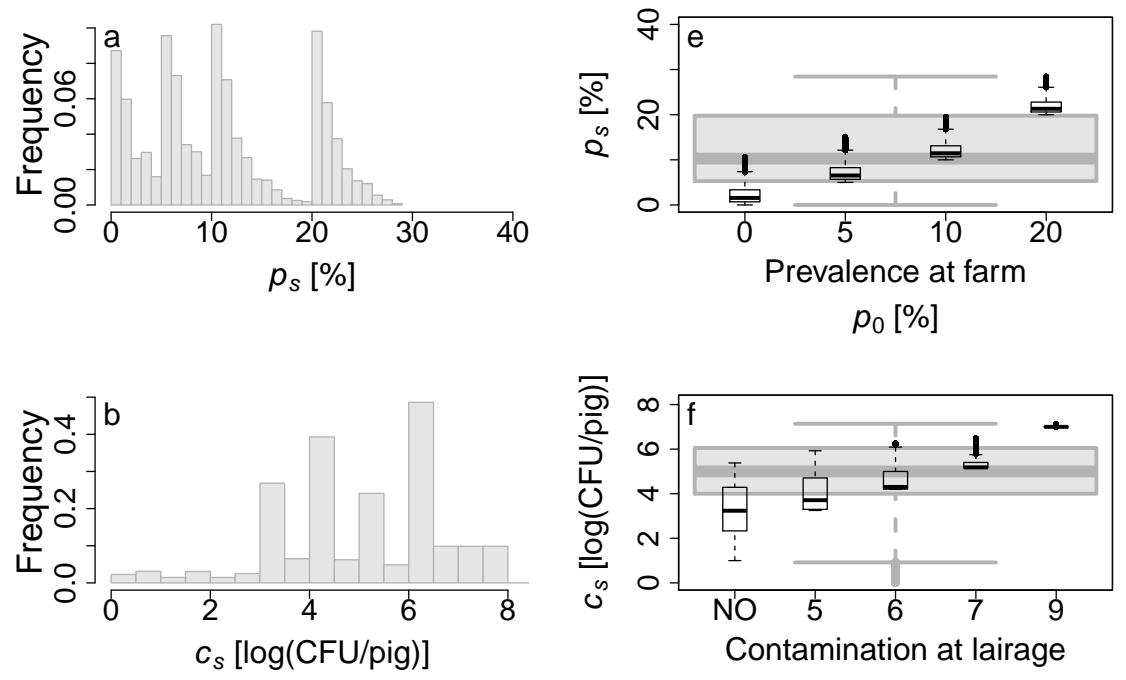

$q_{A}[\log (\mathrm{CFU})]$
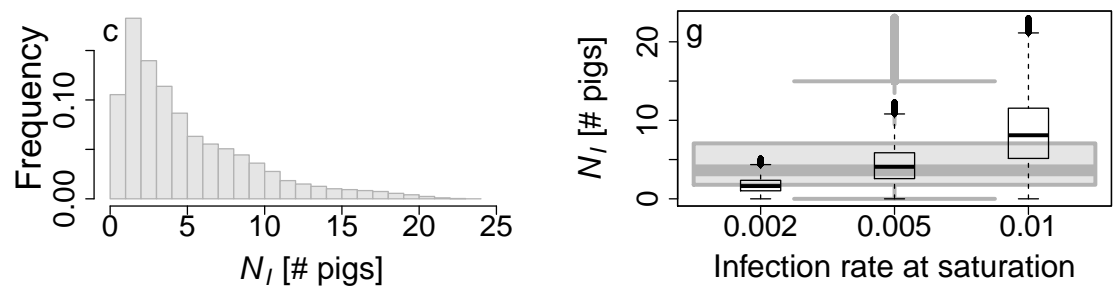

$\pi_{\max }$
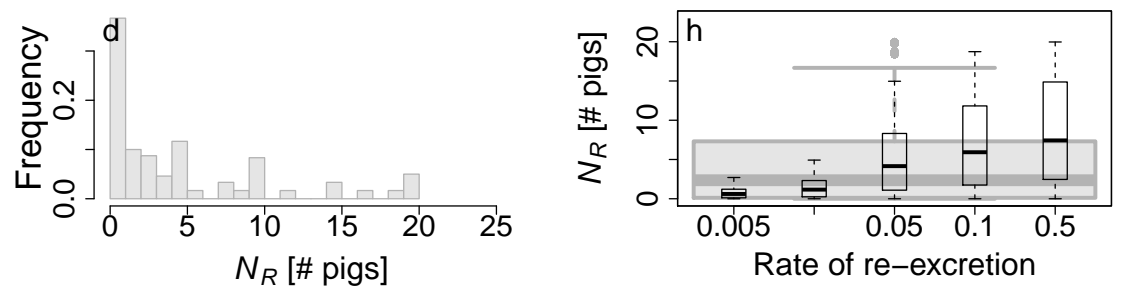

$\rho$

Figure 8: Inter-scenario distributions of the SEIR model outcomes: $(\mathbf{a}, \mathbf{e})$ prevalence at slaughter, $(\mathbf{b}, \mathbf{f})$ average cutaneous contamination at slaughter, $(\mathbf{c}, \mathbf{g})$ new infections from farm to slaughter and $(\mathbf{d}, \mathbf{h})$ number of infected pigs reverting to excretion from farm to slaughter. Histograms $(\mathbf{a}-\mathbf{d})$ present the model outcomes averaged over 501 repetitions for 337500 scenarios. Scenarios cross 9 dose-response functions with 5 excretion rates, 5 reexcretion rates and 1500 management contexts. Corresponding boxplots $(\mathbf{e}-\mathbf{h})$ are presented alongside (grey), as well as boxplots split by the values of the factor that most affects the outcome considered (white): (e) initial prevalence at farm, (f) environmental contamination at lairage, $(\mathrm{g})$ maximum infection probability at saturation (parameter of the dose-response function) and (g) re-excretion rate. 


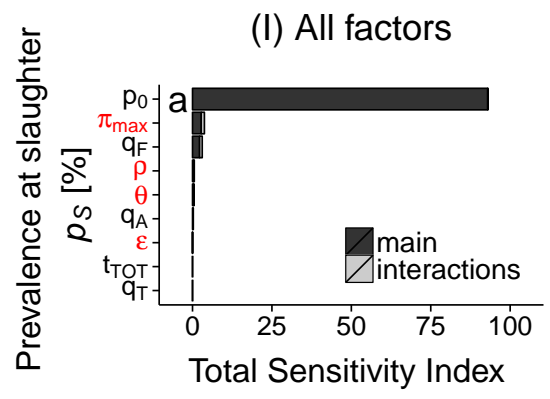

(II) Management factors
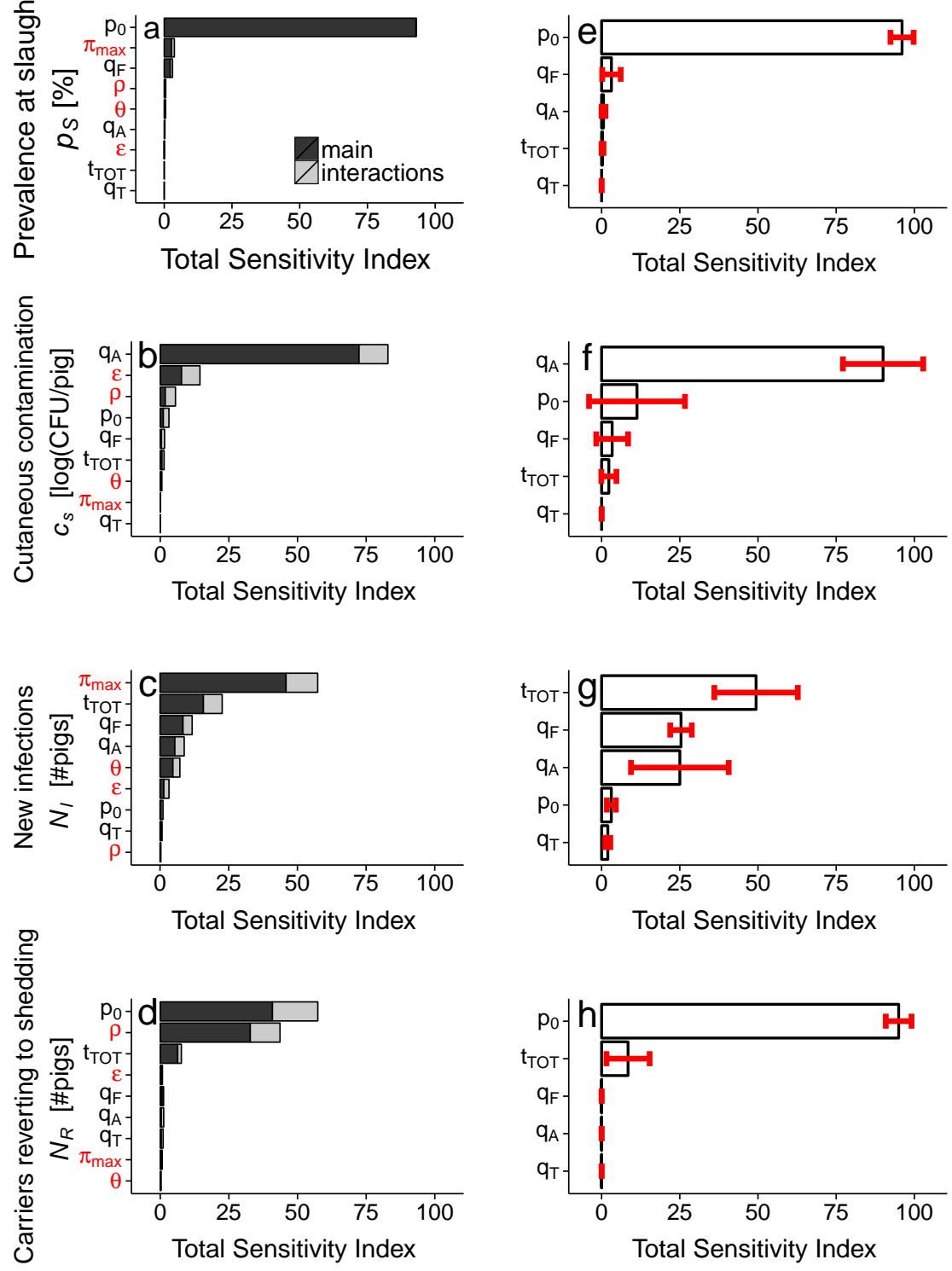

Figure 9: Inter-scenario sensitivity analysis of the of the SEIR model outcomes: (a,e) prevalence at slaughter, $(\mathbf{b}, \mathbf{f})$ average cutaneous contamination at slaughter, $(\mathbf{c}, \mathbf{g})$ new infections from farm to slaughter and $(\mathbf{d}, \mathbf{h})$ number of infected pigs reverting to excretion from farm to slaughter. Outcomes are averaged over 501 repetitions for 337500 scenarios that cross 9 dose-response functions with 5 excretion rates, 5 re-excretion rates and 1500 management contexts. Column (I): total sensitivity indices for all factors, split into main effect (dark grey) and interactions (light grey). Column (II): total sensitivity indices for the management factors alone, averaged over all scenarios sharing the same management constraints; so the associated errorbars represent the variability arising from the uncertainty over the epidemiological parameters. Factors are described in Table 1 
agement factors on outcomes of interest: prevalence at slaughter $\left(p_{s}\right)$, average cutaneous contamination at slaughter $\left(c_{s}\right)$, number of new infections from farm to slaughter $\left(N_{I}\right)$ and number of pigs reverting from carriers to active shedders from farm to slaughter $\left(N_{R}\right)$. The first two outcomes are important to evaluate the risk of carcass contamination in further steps of the pork processing chain. The last two reflect Salmonella circulation. Therefore, they are the effective targets for prevention and control strategies aiming at reducing transmission at this stage of the production chain.

Our model reflects management conditions consistent with the pork production in Brittany, while it considers a great degree of uncertainty over the epidemiological parameters. We evaluated how management and epidemiological factors affect the outcomes at a single batch level, thus focusing on within-batch transmission. Interactions with other batches are represented by the initial environmental contaminations at the farm, in the truck and in the lairage pens. Explicit contamination between batches, together with the analysis of the impact of batch management and cleaning protocols will be addressed in future communications.

The stochastic nature of transmission implies that, under the same scenario 310 (i.e. same management and epidemiological parameters), the model outcomes may vary among repetitions. However, we found that these variations are significantly smaller than the variations observed when changing the scenario. This means that the intra-scenario variability, due to the stochastic nature of transmission, is significantly smaller than the inter-scenario variability, due to the variability in management protocols and the uncertainty in epidemiological parameters. For that reason, we carried out an inter-scenario sensitivity analysis on the simulation outcomes averaged over the intra-scenario repetitions $\left(N_{\text {reps }}=501\right)$. As pigs are not individualised in the model, analysing the variability at the individual level is beyond the scope of this study.

We found that transmission during transport and lairage significantly increases the prevalence and the average cutaneous contamination of the processed batches with $50 \%$ of the scenarios resulting in at least 4 newly-infected pigs and 
$5 \%$ of the scenarios resulting in more than 50 newly-infected pigs (estimates for batches of 100 pigs). It produces new infections and activate non-shedding carriers. This suggests that prevention and control strategies at this stage may prove worthwhile to decrease internal carriage and cutaneous contamination from farm to slaughter.

Such strategies should tackle several management conditions rather than focus on a single aspect of Salmonella spread, because different factors are prevalence at farm, which strongly depends on the herd management and biosecurity (Lurette et al. 2011). Average cutaneous contamination is most affected by the environmental contamination at lairage. Depending on the slaughtering process, pigs may be showered, which should reduce the cutaneous contamigies depends on the transmissibility of the Salmonella strain: for highly transmissible strains, reducing the prevalence at the farm of origin is expected to have the greatest impact on limiting the number of new infections; whereas for less transmissible strains, control measures at lairage should be more effective. and it would be costly to carry it out comprehensively. Moreover, pigs at lairage originate from various farms, so different strains are likely to be present during transport and lairage. Hence, our findings reinforce the idea that interventions 
should occur at each step of the production chain, to robustly reduce Salmonella management from farm to slaughter. 


\section{Acknowledgements}

This work was supported by the French Research Agency (ANR), Program

385

ful to the professional union BDPORC for granting us access to the swine dataset.

\section{References}

BDPORC, 2010, 2010. La base de données professionnelle porcine. URL: http: //www.bdporc.com/defaut/pageInstitution.jsp.

Berriman, A.D.C., Clancy, D., Clough, H.E., Christley, R.M., 2013. Semistochastic models for Salmonella infection within finishing pig units in the 004

Boughton, C., Egan, J., Kelly, G., Markey, B., Leonard, N., 2007. Rapid infection of pigs following exposure to environments contaminated with different levels of Salmonella typhimurium. Foodborne Pathogens and Disease 4, 33-40. doi:10.1089/fpd.2006.58.

EFSA Panel on Biological Hazards (BIOHAZ), 2010. Scientific opinion on a quantitative microbiological risk assessment of Salmonella in slaughter and breeder pigs. EFSA Journal 8, 1547. doi 10.2903/j.efsa.2010.1547

European Food Safety Authority, 2008. Report of the Task Force on Zoonoses Data Collection on the Analysis of the baseline survey on the prevalence of Salmonella in slaughter pigs, in the EU, 2006-2007 [1] - Part A: Salmonella prevalence estimates. EFSA Journal 6. doi $10.2903 /$ j.efsa.2008.135r.

Fekedulegn, D., Mac Siurtain, M.P., Colbert, J.J., 1999. Parameter estimation q of nonlinear growth models in forestry. Silva Fennica 33, 327-336. doi 10. $14214 /$ sf. 653 
Frotin, P., Gault, E., Chevillon, P., Eon, Y., 2007. Mise à jour des références techniques et des recommandations sur le process dabattage dans 15 abatn toirs. Rapport d'étude. IFIP (France). URL: http://www.ifip.asso.fr/ fr/documentations?nid=9182417.

Hald, T., Wingstrand, A., Swanenburg, M., von Altrock, A., Thorberg, B.M., 2003. The occurrence and epidemiology of Salmonella in European pig

415 „ slaughterhouses. Epidemiology and Infection 131, 1187-1203. doi 10.1017/ S0950268803001171.

Hernández, M., Gómez-Laguna, J., Luque, I., Herrera-León, S., Maldonado, A., Reguillo, L., Astorga, R.J., 2013. Salmonella prevalence and characterization in a free-range pig processing plant: tracking in trucks, lairage, slaughter line and quartering. International Journal of Food Microbiology 162, 48-54. doi:10.1016/j.ijfoodmicro.2012.12.026.

Hill, A.A., Simons, R.R.L., Kelly, L., Snary, E.L., 2015. A farm transmission model for Salmonella in pigs, applicable to EU member states. Risk Analysis doi:10.1111/risa.12356

${ }_{425}$ Hotes, S., Traulsen, I., Krieter, J., 2012. An individual-based model for Salmonella transmission along the pig production chain. Archiv Tierzucht

q - Archives Animal Breeding 55, 48-63. URL: http://www.archanimbreed. com/content/2012/abs12_01.html\#A5

Hurd, H.S., Gailey, J.K., McKean, J.D., Rostagno, M.H., 2001a. Rapid infection in market-weight swine following exposure to a Salmonella Typhimuriumcontaminated environment. American Journal of Veterinary Research 62, 1194-1197. doi $10.2460 / a j v r .2001 .62 .1194$

Hurd, H.S., McKean, J.D., Griffith, R.W., Wesley, I.V., Rostagno, M.H., 2002. Salmonella enterica infections in market swine with and without transport 435 a and holding. Applied and Environmental Microbiology 68, 2376-2381. doi 10. 1128/AEM.68.5.2376-2381.2002. 
Hurd, H.S., McKean, J.D., Wesley, I.V., Karriker, L.A., 2001b. The effect of lairage on Salmonella isolation from market swine. Journal of Food Protection 64, 939-944. URL: http://www.ingentaconnect.com/content/iafp/jfp/ 2001/00000064/00000007/art00003

Ivanek, R., Österberg, J., Gautam, R., Sternberg Lewerin, S., 2012. Salmonella fecal shedding and immune responses are dose- and serotype- dependent in pigs. PLoS ONE 7, e34660. doi:10.1371/journal.pone.0034660

Lamboni, M., Monod, H., Makowski, D., 2011. Multivariate sensitivity analysis to measure global contribution of input factors in dynamic models. Reliability ㅁ Engineering \& System Safety 96, 450-459. doi 10.1016/j.ress.2010.12. 002 .

Loynachan, A.T., Harris, D.L., 2005. Dose determination for acute Salmonella infection in pigs. Applied and Environmental Microbiology 71, 2753-2755. doi:10.1128/AEM.71.5.2753-2755.2005.

Lurette, A., Belloc, C., Touzeau, S., Hoch, T., Ezanno, P., Seegers, H., Fourichon, C., 2008. Modelling Salmonella spread within a farrow-to-finish pig herd. Veterinary Research 39, 49. doi 10.1051/vetres:2008026.

Lurette, A., Touzeau, S., Ezanno, P., Hoch, T., Fourichon, C., Seegers, H., Belloc, C., 2011. Within-herd biosecurity and Salmonella seroprevalence in slaughter pigs: a simulation study. Journal of Animal Science 89, 2210-2219. doi:10.2527/jas.2010-2916.

Mannion, C., Fanning, J., McLernon, J., Lendrum, L., Gutierrez, M., Duggan, S., Egan, J., 2012. The role of transport, lairage and slaughter processes in the dissemination of Salmonella spp. in pigs in Ireland. Food Research International 45, 871-879. doi:10.1016/j.foodres.2011.02.001.

Martín-Peláez, S., Peralta, B., Creus, E., Dalmau, A., Velarde, A., Pérez, J.F., Mateu, E., Martín-Orúe, S.M., 2009. Different feed withdrawal times before slaughter influence caecal fermentation and faecal Salmonella shedding in 
${ }_{465}$ pigs. The Veterinary Journal 182, 469-73. doi $10.1016 / j . t v j 1.2008 .08$. 002 .

Pires, S.M., Hald, T., 2010. Assessing the differences in public health impact of Salmonella subtypes using a Bayesian microbial subtyping approach for

n source attribution. Foodborne Pathogens and Disease 7, 143-151. doi 10. $1089 /$ fpd.2009.0369

Rostagno, M.H., Callaway, T.R., 2012. Pre-harvest risk factors for Salmonella

घ enterica in pork production. Food Research International 45, 634-640. doi 10. $1016 /$ j.foodres.2011.04.041.

Rostagno, M.H., Hurd, H.S., McKean, J.D., Ziemer, C.J., Gailey, J.K., Leite, R.C., 2003. Preslaughter holding environment in pork plants is highly contaminated with Salmonella enterica. Applied and Environmental Microbiology 69, 4489-4494. doi 10.1128/AEM.69.8.4489-4494.2003.

Saltelli, A., Tarantola, S., Campolongo, F., 2000. Sensitivity analysis as 口 an ingredient of modeling. Statistical Science 15, 377-395. URL: http: //projecteuclid.org/euclid.ss/1009213004.

Schaffner, D.W., Doyle, M.P. (Eds.), 2008. Microbial Risk Analysis of Foods.

n Emerging Issues in Food Safety, American Society of Microbiology. doi 10. $1128 / 9781555815752$.

Scherer, K., Szabó, I., Rösler, U., Appel, B., Hensel, A., Nöckler, K., 2008. Time course of infection with Salmonella typhimurium and its influence on fecal shedding, distribution in inner organs, and antibody response in fattening pigs. Journal of Food Protection 71, 699-

a 705. URL: http://www.ingentaconnect.com/content/iafp/jfp/2008/ $00000071 / 00000004 /$ art00004.

490 Simons, R.R.L., Hill, A.A., Swart, A., Kelly, L., Snary, E.L., 2015. A transport and lairage model for Salmonella transmission between pigs applicable to EU member states. Risk Analysis doi $10.1111 /$ risa.12390 
Small, A., James, C., James, S., Davies, R., Liebana, E., Howell, M., Hutchison, M., Buncic, S., 2006. Presence of Salmonella in the red meat abattoir lairage after routine cleansing and disinfection and on carcasses. Journal of

Food Protection 69, 2342-2351. URL: http://www.ingentaconnect.com/ content/iafp/jfp/2006/00000069/00000010/art00004.

Smid, J.H., Heres, L., Havelaar, A.H., Pielaat, A., 2012. A biotracing model of Salmonella in the pork production chain. Journal of Food Protection 75, 270-280. doi:10.4315/0362-028X. JFP-11-281.

Swanenburg, M., Urlings, H.A.P., Keuzenkamp, D.A., Snijders, J.M.A., 2001. Salmonella in the lairage of pig slaughterhouses. Journal of Food Protecq tion 64, 12-16. URL: http://www.ingentaconnect.com/content/iafp/ jfp/2001/00000064/00000001/art00002.

${ }_{505}$ Tanaka, T., Imai, Y., Kumagae, N., Sasaki, T., Ochiai, N., Uruno, K., Kitazawa, H., Saito, T., Sato, S., 2014. Quantitative microbiological evaluation of Salmonella typhimurium shed in diarrhea, loose, and normal stools of infected pigs. Open Journal of Veterinary Medicine 4, 58-66. doi:10.4236/ojvm.2014.44007.

VLA, DTU, RIVM, 2010. Quantitative Microbiological Risk Assessment on Salmonella in Slaughter and Breeder pigs: Final Report. External scientific report produced for EFSA (Question No EFSA-Q-2007-00245). VLA (Veterinary Laboratories Agency, UK) in consortium with DTU (Danish Technical University, Denmark) and RIVM (Rijksinstituut Volksgezondheid en Milieu,

515 The Netherlands). URL: http://www.efsa.europa.eu/fr/supporting/ pub/46e.htm. 